Draft Version June 24, 2021

Typeset using $\mathrm{LAT}_{\mathrm{E}} \mathrm{X}$ modern style in AASTeX61

\title{
OBSERVATIONAL EVIDENCE FOR SELF-GENERATION OF SMALL-SCALE MAGNETIC FLUX ROPES FROM INTERMITTENT SOLAR WIND TURBULENCE
}

\author{
JINLEI ZHENG ${ }^{1}$ AND QIANG HU ${ }^{2}$ \\ ${ }^{1}$ Department of Space Science \\ The University of Alabama in Huntsville \\ Huntsville, AL 35805, USA \\ ${ }^{2}$ Department of Space Science and CSPAR \\ The University of Alabama in Huntsville \\ Huntsville, AL 35805, USA
}

(Received 15 November 2017; Revised 20 December 2017; Accepted 22 December 2017)

Submitted to ApJL

\begin{abstract}
We present unique and additional observational evidence for the self-generation of small-scale coherent magnetic flux rope structures in the solar wind. Such structures with durations between 9 and 361 minutes are identified from Wind in-situ spacecraft measurements through the Grad-Shafranov (GS) reconstruction approach. The event occurrence counts are on the order of 3,500 per year on average and have a clear solar cycle dependence. We build a database of small-scale magnetic flux ropes from twenty-year worth of Wind spacecraft data. We show a power-law distribution of the wall-to-wall time corresponding well to the inertial range turbulence, which agrees with relevant observation and numerical simulation results. We also provide the axial current density distribution from the GS-based observational analysis which yields non-Gaussian probability density function consistent with numerical simulation result.
\end{abstract}

Keywords: magnetic flux ropes — Grad-Shafranov — turbulence solar wind

Corresponding author: Qiang Hu qiang.hu.th@dartmouth.edu, qh0001@uah.edu 


\section{INTRODUCTION}

Small-scale magnetic flux ropes in the solar wind of durations ranging from a few minutes to a few hours have been identified from in-situ spacecraft data and studied for decades (e.g., Moldwin et al. 1995; Moldwin et al. 2000; Cartwright \& Moldwin 2010; Feng et al. 2008; Yu et al. 2014). They possess some similar features in magnetic field configurations to their large-scale counterparts, the magnetic clouds (MCs) of durations lasting for a dozen hours up to a few days, but differ in certain plasma properties. Unlike MCs which have a clear solar origin related to coronal mass ejections (CMEs), the origin of these small-scale magnetic flux ropes is still debated. One view is that they also have a solar source correspondence, especially for intermediatesized flux ropes (e.g., Feng et al. 2008), based on some similar statistical properties as MCs. As advocated by Borovsky (2008), the "flux tubes" bounded by discontinuities may be rooted on the Sun, permeating the whole interplanetary space. The other view, supported not only by observational analysis, but also extensively by numerical simulations over a wide range of scales (Servidio et al. 2008; Greco et al. 2008, 2009a,b; Greco et al. 2010; Wan et al. 2013, 2015), states that the generation process of small-scale magnetic flux ropes or islands in strict two-dimensional (2D) configuration is intrinsic to magnetohydrodynamic (MHD) turbulence often approximated by a quasi-2D model or containing a dominant 2D component (e.g., Matthaeus et al. 2007; Zank et al. 2017, and references therein). They are believed to be the byproduct of solar wind turbulence dynamic evolution process, resulting in the generation of coherent structures including "small random current", "current cores" and "current sheets" (Greco et al. 2009a) over the inertial range length scales.

Accompanying these studies associated with small-scale flux ropes, the observational analysis on the discontinuities or current sheets possibly bounding the flux ropes (or flux tubes; see, e.g., Borovsky (2008)) is also ongoing. Various approaches have been utilized to identify these structures mostly as incremental changes in magnetic field from in-situ time-series data (e.g., Bruno et al. 2001; Vasquez et al. 2007; Borovsky 2008; Greco et al. 2009a; Greco et al. 2010; Miao et al. 2011; Osman et al. 2014; Greco et al. 2016). However there generally lacks a synergy between the analyses of these two types of coherent structures, and the analysis method for the smallscale magnetic flux ropes is outdated. In this Letter, we explore the application of the Grad-Shafranov (GS) reconstruction technique to the automated detection of smallscale magnetic flux ropes for the first time. Meanwhile, we report on the successful identification of an unprecedented number of the small-scale flux rope events via the GS approach and associated analysis results including unique physical characterization of these structures, especially the axial current density distribution, which enables a direct comparison with the numerical simulation results for 2D MHD turbulence. To the best of our knowledge, the estimate of current density has to be achieved by using closely-spaced multiple spacecraft through the curlometer approach, such as from the Cluster and Magnetospheric Multiscale (MMS) missions (see, e.g., Greco et al. 
2018, for such a comparison of current density between MMS measurements and 2D simulations).

The GS reconstruction technique is based on the GS equation, describing 2D (or $2.5 \mathrm{D}$ with non-vanishing axial magnetic field) cylindrical magnetic field and plasma configurations in magnetohydrostatic equilibrium (see Hu (2017) for a comprehensive review) that is more general than the force-free assumption. For the magnetic field components $\mathbf{B}=\left(\partial A / \partial y,-\partial A / \partial x, B_{z}(A)\right)$ defined by the $2 \mathrm{D}$ magnetic flux function $A(x, y)$ in a Cartesian coordinates (with $z$-axis along the flux rope axis, and $\partial / \partial z \approx 0$ ), the transverse force balance yields the GS equation (reduced from the usual equation $\nabla p=\mathbf{J} \times \mathbf{B})$,

$$
\frac{\partial^{2} A}{\partial x^{2}}+\frac{\partial^{2} A}{\partial y^{2}}=-\mu_{0} \frac{d P_{t}}{d A}=-\mu_{0} J_{z}(A) .
$$

Here the right-hand side gives the axial current density $J_{z}$ which is a total derivative of the transverse pressure $P_{t}=p+B_{z}^{2} / 2 \mu_{0}$, the sum of the plasma pressure and the axial magnetic pressure, with respect to $A$. All these quantities can be evaluated along a single-spacecraft path across a flux rope structure. Since the flux function $A$ characterizes the nested cylindrical flux surfaces of a flux rope, the axial current density distribution throughout such a flux rope configuration is readily obtained by the function $J_{z}(A)$ determined from in-situ spacecraft data. Additionally the cross section of a cylindrical flux rope given by the solution $A(x, y)$ to the GS equation over a rectangular domain can also be obtained numerically (Hu \& Sonnerup 2001; $\mathrm{Hu} \&$ Sonnerup 2002). The technique has been widely applied to reconstruct structures in a variety of space plasma regimes (see, e.g., Hu 2017). The application of the GS method to the small-scale structures of relevance to the quasi-2D MHD turbulence as envisaged by Matthaeus et al. (2007) has just begun.

Telloni et al. (2016) took yet another approach by evaluating the MHD rugged invariants (see also, Telloni et al. 2013) within about 144 flux ropes identified in prior studies. They concluded that flux ropes represent well-organized structures coming from the dynamical evolution of MHD turbulent cascade, in which the MHD invariants are inter-related. They further stated that the flux ropes dynamically evolve toward a final steady state in which the (normalized) magnetic helicity $\sigma_{m}$, and cross-helicity $\sigma_{c}$ values within the structures are distributed according to $\sigma_{m}^{2}+\sigma_{c}^{2}=1$. We expect to complement that study by providing a more exhaustive list of events equipped with more comprehensive characterizations of flux rope properties. For instance, from the GS reconstruction output, we will be able to derive, quantitatively and directly, the total magnetic energy, magnetic flux, and (relative) magnetic helicity ( $\mathrm{Hu} 2017$ ) contained in each flux rope, although this is not the focus of the current analysis, and we have excluded Alfvénic structures or structures with high Alfvénicity.

We present and introduce briefly the small-scale flux rope database built by an automated process based on the GS method in the next section. In Section 3, we present the analysis results of the wall-to-wall time and the axial current density dis- 
tributions derived from our database and compare directly with Greco et al. (2009a). We conclude in the last section, and signify the uniqueness of our approach and result in support of the view of the self-generation of small-scale flux ropes via MHD turbulence.

\section{SMALL-SCALE FLUX ROPE DATABASE VIA THE GS RECONSTRUCTION METHOD}

We have built a small-scale magnetic flux rope database via the GS reconstruction method. The GS method was first applied to reconstruct cross sections of smallscale magnetic flux ropes in the solar wind with durations of about half an hour in Hu \& Sonnerup (2001). In the present study, we apply to Wind in-situ spacecraft measurements of 1-minute cadence between 1996 and 2016, based on largely the same principles and procedures without carrying out the final numerical reconstruction of solving for a 2D solution to the GS equation on the cross-sectional plane. Detailed descriptions of the automated detection algorithm including a flowchart illustrating the step-by-step implementation of the GS-based algorithm are presented in Zheng (2017).

Table 1. Metrics and Selection Criteria for the GS-based Automated Flux Rope Detection Algorithm

\begin{tabular}{ccccc}
\hline Duration (minutes) & $\bar{B}(\mathrm{nT})$ & $R E S$ & $R_{f}$ & Walén slope \\
\hline $9 \sim 361$ & $\geq 5$ & $\leq 0.12$ & $\leq 0.14$ & $\leq 0.3$ \\
\hline
\end{tabular}

In short, the detection procedures start by sliding a rectangular window of a chosen width through the time series to select the data interval for analysis. For each interval and the selected magnetic field and plasma parameters, the same procedures apply as the standard GS reconstruction of 2.5D magnetohydrostatic structures. The main steps are to obtain the flux function $A$ and the transverse pressure $P_{t}$ values along the spacecraft path in a properly determined trial frame of reference (Hu \& Sonnerup 2002). Then the necessary conditions for a $2.5 \mathrm{D}$ cylindrical magnetic flux rope configuration, mainly the requirement that the function $P_{t}(A)$ be single-valued based on the GS equation and the double-folding pattern in $A$ across the flux rope characteristic of nested closed flux surfaces (each of a distinct $A$ value), are checked by producing a set of quantitative metrics. After a trial-and-error process, especially by enumerating all the possible $z$-axis orientations in its parameter space, the set of metrics is obtained and a flux rope candidate is positively identified if the selection threshold conditions are satisfied, as indicated in Table 1. The flux rope interval is then identified and recorded with a size/duration limited by the width of the data window. Flux ropes with different sizes are simply identified by iterations, repeating the aforementioned procedures with different-width sliding windows. Additional post-processing procedures are taken to clean up overlapping intervals and to ensure good-quality events 


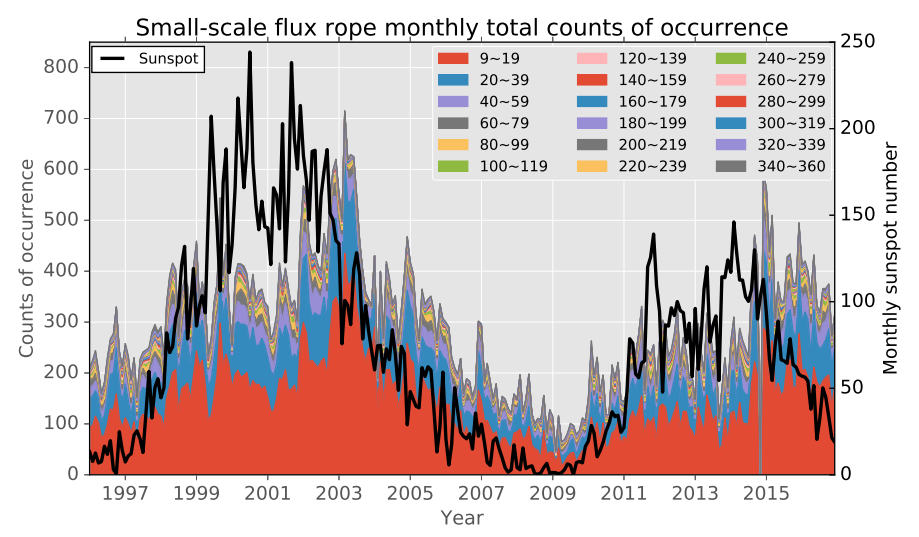

Figure 1. Monthly counts (left axis) of small-scale magnetic flux rope events identified from Wind in-situ spacecraft measurements during 1996-2016. The counts are separated for flux ropes of different durations and color-coded as indicated by the legends in minutes. The corresponding monthly sunspot numbers are given by the black curve (right axis).

in our database according to the additional metrics in Table 1. In summary, the two metrics, RES and $R_{f}$, originally defined in Hu \& Sonnerup (2002) and Hu et al. (2004), respectively, evaluate the quality of $P_{t}(A)$ being single-valued and each has to be smaller than the specific threshold. The Walén slope (Paschmann \& Sonnerup 2008), corresponding to the average ratio between the remaining plasma flow (ideally zero) in the frame of reference moving with the flux rope and the local Alfvén velocity, is used as a criterion to remove Alfvénic structures. We also have the option to restrict our database to only contain flux ropes of average magnetic field magnitude $\bar{B} \geq 5 \mathrm{nT}$, following the previous studies (e.g., Cartwright \& Moldwin 2010) and avoiding complications due to low Alfvén speed, in the present study. Therefore, our database contains the small-scale flux ropes with helical magnetic field configuration and negligible remaining plasma flow, corresponding to $\sigma_{c} \approx 0$ and the maximum $\sigma_{m} \approx \pm 1$ as expected based on Telloni et al. (2016). However our database contains events with a wide range of plasma (proton) $\beta$ values, yielding both a mean and a median around 0.5 , since we are not limited to finding low- $\beta$ structures only.

We have identified a total number of 74,241 distinct small-scale magnetic flux rope events with durations ranging from 9 to 361 minutes from the Wind spacecraft data sets. We have summarized and compiled our detection results onto a publicly available website, http://fluxrope.info, which contains some essential information about the properties of the detected flux rope structures. Various statistical analyses have been performed and reported in Zheng (2017). At a glimpse, Figure 1 presents the monthly event counts in our database, covering the past two solar cycles. Clearly the total counts including all events of variable durations follow the monthly sunspot numbers, hinting at solar-cycle dependency of their occurrence. The events of smaller durations generally have greater rates of occurrence. The peaks of occurrence counts tend to appear in the declining phase of each solar cycle. On average, we have identified more than 3,500 small-scale magnetic flux ropes per year. We caution, however, 


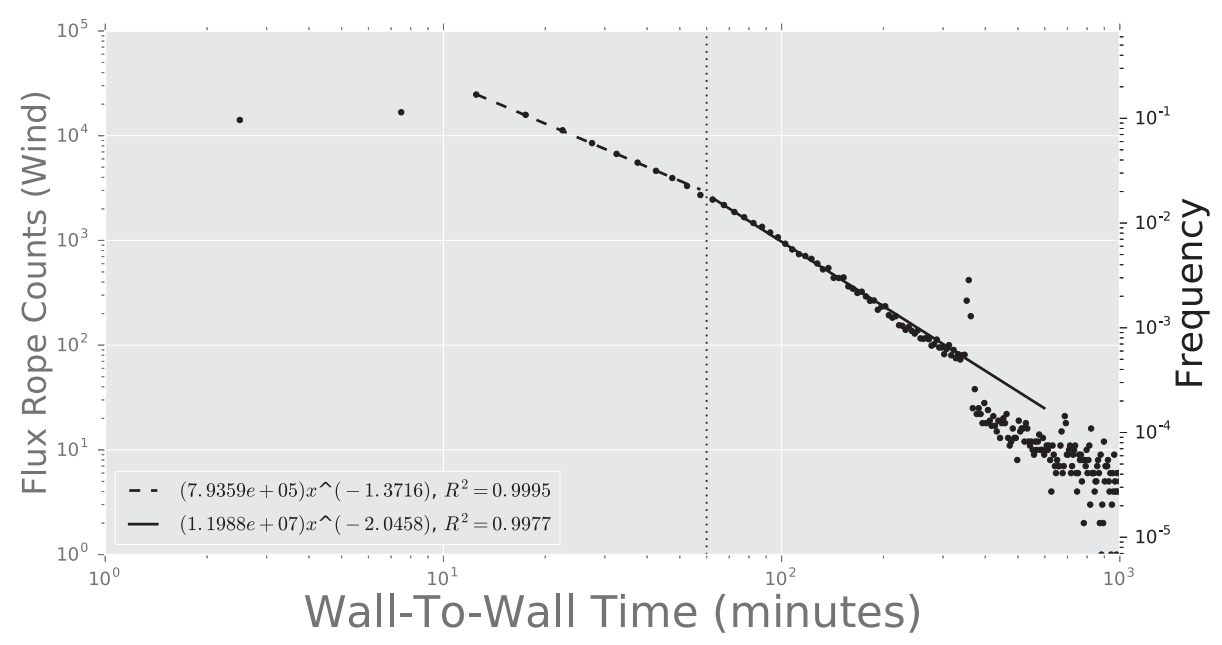

Figure 2. The wall-to-wall time distribution of small-scale flux ropes in our database. The dashed and solid black lines are the power-law fittings to the two sections divided by the dotted line at 60 minutes. The fitting parameters are denoted, for each line, in the lower left corner with the goodness-of-fit parameter $R^{2}$, the coefficient of determination.

against a quick conclusion about the solar origin of these structures, because we believe there should be a distinction between "solar origin" and "solar activity dependence" as indicated by the dependence on sunspot numbers for the latter. In addition, some local turbulence processes could also be modulated by the solar activity, leading to the solar-cycle dependence as exhibited here. For example, perhaps the simplest case was the modulation of cosmic ray transport coefficients by the solar-cycle variations in the magnetic field magnitude and variance (Manuel et al. 2011). Furthermore, the recent works of Zhao et al. (2017, 2018) showed the solarcycle dependence of various turbulence quantities affecting cosmic ray diffusion, such as Elsässer variables, correlation lengths, and residue energy etc. They are all derived locally and with contributions from local driving sources, based on sophisticated MHD turbulence theory (Zank et al. 2017; Adhikari et al. 2014).

\section{WALL-TO-WALL TIME AND AXIAL CURRENT DENSITY DISTRIBUTIONS}

With such a small-scale magnetic flux rope database, we are positioned to perform detailed statistical analysis in addition to the occurrence rate distribution demonstrated in Figure 1. We report here important findings about the wall-to-wall time and the axial current density $J_{z}$ distributions from the identified flux ropes. They have direct and significant relevance to the findings of Greco et al. (2009a).

The results from our analysis are presented in Figures 2 and 3. The current density is derived from $d P_{t} / d A$, as indicated in equation (1), where both $P_{t}$ and $A$ are evaluated from in-situ spacecraft measurements for each individual event. To facilitate a direct comparison with the numerical simulation result which is $2 \mathrm{D}$ in nature (Greco et al. 2009a), we calculated the axial current density samples in each event at a rate proportional to its scale size and congregated the results together from all events. 
The flux rope wall-to-wall time is simply the distribution by putting together the flux rope duration and the separation time in-between adjacent flux rope intervals. The flux rope wall or boundary in our database is considered as a type of discontinuity (see, e.g., Borovsky 2008) or current sheet. Therefore the wall-to-wall time in our analysis is used as a proxy to the waiting time between current sheets or discontinuities of negligible thicknesses. Such waiting time distributions (WTDs) have been analyzed in a number of previous works (e.g., Bruno et al. 2001; Vasquez et al. 2007; Greco et al. 2008, 2009a,b; Miao et al. 2011) by directly identifying current sheets or discontinuities from in-situ time-series data. We note that our proxy is unique and valid for flux ropes bounded by discontinuities broadly defined as locations where the magnetic field and/or plasma parameters change. This is consistent with our choice of flux rope boundaries based on the GS method (Hu et al. 2004). They are chosen as the locations along the approximately single-valued and double-folded $P_{t}$ versus $A$ curve, corresponding to the specific data points in the time series of both the magnetic field and plasma measurements. Beyond those points, i.e., beyond the flux rope boundary, the data, in terms of $P_{t}=p+B_{z}^{2} / 2 \mu_{0}$ as a function of $A$, start to deviate. This often corresponds to the concurrent changes or increments in magnetic field and plasma parameters when a flux-tube (or rope) boundary is encountered (Borovsky 2008). Therefore this lends to the justification for using the wall-to-wall time as a proxy to the current sheet waiting time.

Figure 2 shows the wall-to-wall time distribution of small-scale flux ropes. The distribution is well fitted by two power law functions of different power indices as indicated, with a break point located at $\sim 60$ minutes. The outliers near the two ends are due to cutoff effect of limited durations for these events. The WTDs of the observed solar wind discontinuities in Greco et al. (2009a) showed a power law within the break point at $\sim 50$ minutes, corresponding to the typical correlation length scale (Matthaeus et al. 2005), which is consistent with our result. In addition, the power law index from Greco et al. (2009b) is $-0.92 \pm 0.03$ from numerical simulation, and $-1.23 \pm 0.03$ from the in-situ spacecraft observations at $1 \mathrm{AU}$, for the range below the break point, i.e., in the inertial range of solar wind turbulence. They compare well with our result, -1.37 for the dashed black line in Figure 2. We note that for the case including additional flux rope events by relaxing the criterion on $\bar{B}$ to $\bar{B}>0$, the number of events doubles and the power law fitting to the wall-to-wall time persists. The index for the inertial range becomes -1.22 . The section beyond the break point can still be fitted by a power law function with a power index $\sim-2$. However the interpretation of this portion is not certain and has yet to be improved with better statistics.

Figure 3 shows the PDF of the normalized axial current density distribution inside and near the boundaries of the small-scale magnetic flux ropes in our database, in the same format as the result presented by Greco et al. (2009a) from 2D MHD simulation. They also characterized their non-Gaussian PDF by three regions: regions I and III 


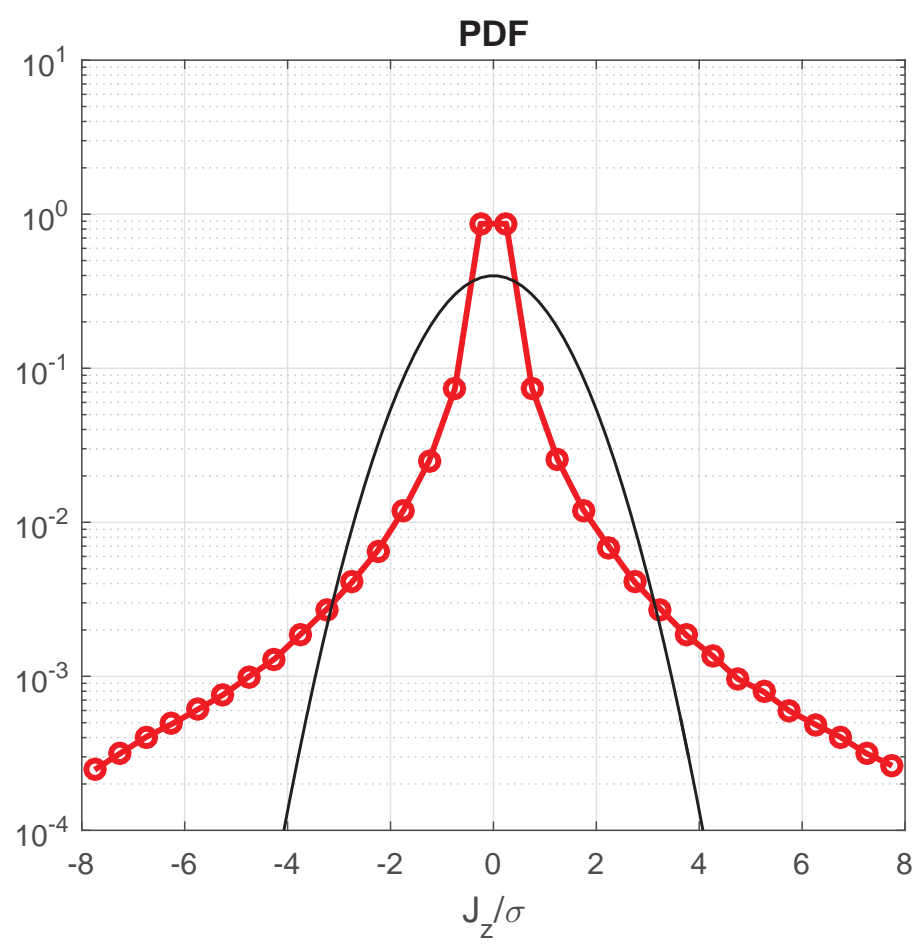

Figure 3. The probability density function (PDF) of the axial current density $J_{z}$ distribution associated with magnetic flux ropes normalized by the standard deviation $\sigma$ $\left(\sigma=8.42 \times 10^{-11} \mathrm{~A} / \mathrm{m}^{2}\right)$. The thin black curve is the standard Gaussian distribution with unit variance.

are super-Gaussian, around the center and toward the tails of the PDF, corresponding to current-sheet like structures in-between magnetic flux ropes or magnetic islands, but with the weakest and strongest current density in magnitude, respectively; region II corresponds to the sub-Gaussian section with modest magnitude of current density, mostly appearing inside flux rope cores. Our result in Figure 3 exhibits the same general non-Gaussian features in agreement with Greco et al. (2009a).

\section{CONCLUSIONS}

In conclusion, in this Letter, we report the analysis result on the wall-to-wall time and axial current density distributions from in-situ spacecraft observations of smallscale magnetic flux ropes based on an extensive event database and the unique GS model output. Our results show a power-law distribution of the wall-to-wall time for length scales smaller than the correlation length, corresponding well to the inertial range of solar wind turbulence. This result is consistent with the analysis result of Greco et al. (2009a) from both observational analysis using an entirely different approach and MHD simulations of intermittent turbulence. In addition, we also obtain the non-Gaussian distribution of the axial current density associated with the magnetic flux ropes, which is also in agreement with the numerical simulation result of Greco et al. (2009a). We therefore conclude that we have provided unique and additional observational evidence in support of the view of self-generation of coherent 
structures, such as small-scale magnetic flux ropes and current sheets, locally from MHD turbulence. In light of the analysis by Telloni et al. (2016), we plan to extend our analysis to include structures of significant remaining flow, i.e., structures with $\sigma_{c} \neq 0$. This is feasible since the extension of the GS method to the GS-type with significant field-aligned flow and even to the 2D MHD equilibrium has been developed (see Hu 2017). Meanwhile we also invite other researchers to extend their relevant studies by utilizing our extensive database.

We are grateful to our colleagues, Drs. Laxman Adhikari, Jakobus le Roux, Gang Li, Gary Webb, Gary Zank, and Lingling Zhao for illuminating discussions and suggestions that have made this work possible. We acknowledge NASA grants NNX12AH50G, NNX14AF41G, NNX17AB85G, subawards NRL N00173-141-G006 and SAO SV4-84017, and NSF grant AGS-1650854 for support. We thank Drs. M. G. Linton and P. Riley for stimulating discussions on the GS-based flux rope identification which initiated this work. We also thank the reviewer for his/her expert comments and suggestions.

\section{REFERENCES}

Adhikari, L., Zank, G. P., Hu, Q., \&

Dosch, A. 2014, ApJ, 793, 52

Borovsky, J. E. 2008, Journal of

Geophysical Research (Space Physics), 113, A08110

Bruno, R., Carbone, V., Veltri, P.,

Pietropaolo, E., \& Bavassano, B. 2001,

Planetary Space Science, 49, 1201

Cartwright, M. L., \& Moldwin, M. B. 2010, Journal of Geophysical Research (Space Physics), 115, A08102, a08102. http://dx.doi.org/10.1029/2009JA014271Greco, A., Servidio, S., Matthaeus, W. H.,

Feng, H. Q., Wu, D. J., Lin, C. C., et al. 2008, Journal of Geophysical Research: Space Physics, 113, A12105, a12105. http://dx.doi.org/10.1029/2008JA013103

Greco, A., Chuychai, P., Matthaeus, W. H., Servidio, S., \& Dmitruk, P. 2008, Geophysical Research Letters, 35, doi:10.1029/2008GL035454, 119111. http://dx.doi.org/10.1029/2008GL035454

Greco, A., Matthaeus, W. H., Perri, S., et al. 2018, SSRv, 214, 1
Greco, A., Matthaeus, W. H., Servidio, S., Chuychai, P., \& Dmitruk, P. 2009a, The Astrophysical Journal Letters, 691, L111. http://stacks.iop.org/1538-4357/691/i=2/a=L111

Greco, A., Matthaeus, W. H., Servidio, S., \& Dmitruk, P. 2009b, Phys. Rev. E, 80, 046401. https://link.aps.org/doi/10.1103/PhysRevE.80.0464

Greco, A., Perri, S., Servidio, S., Yordanova, E., \& Veltri, P. 2016, ApJL, 823, L39 \& Dmitruk, P. 2010, Planet. Space Sci., 58, 1895

Hu, Q. 2017, Science China Earth Sciences, 60, 1466. https://doi .org/10.1007/s11430-017-9067-2

Hu, Q., Smith, C. W., Ness, N. F., \& Skoug, R. M. 2004, Journal of Geophysical Research: Space Physics, 109, doi:10.1029/2003JA010101, a03102. http://dx.doi.org/10.1029/2003JA010101

Hu, Q., \& Sonnerup, B. U. Ö. 2001, Geophysical Research Letters, 28, 467 
Hu, Q., \& Sonnerup, B. U. O. 2002, Journal of Geophysical Research: Space Physics, 107, SSH 10. http://dx.doi.org/10.1029/2001JA000293 Telloni, D., Carbone, V., Perri, S., et al.

Manuel, R., Ferreira, S., Potgieter, M., Strauss, R., \& Engelbrecht, N. 2011, Advances in Space Research, 47, 1529 .

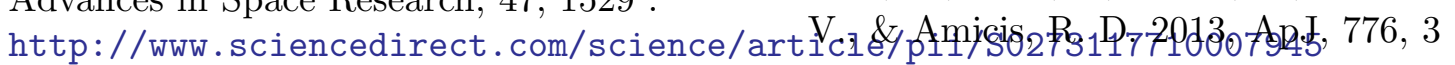

Matthaeus, W. H., Bieber, J. W., Ruffolo, D., Chuychai, P., \& Minnie, J. 2007, ApJ, 667, 956

Matthaeus, W. H., Dasso, S., Weygand, J. M., et al. 2005, Phys. Rev. Lett., 95, 231101. https://link.aps.org/doi/10.1103/PhysRe

Miao, B., Peng, B., \& Li, G. 2011, Annales Geophysicae, 29, 237. https : //www . ann-geophys . net/29/237/2011/

Moldwin, M. B., Ford, S., Lepping, R., Slavin, J., \& Szabo, A. 2000, Journal of Geophysical Research: Space Physics, 27,57

Moldwin, M. B., Phillips, J. L., Gosling, J. T., et al. 1995, Journal of Geophysical Research: Space Physics, 100, 19903. http://dx.doi.org/10.1029/95JA01123

Osman, K. T., Matthaeus, W. H., Gosling, J. T., et al. 2014, Physical Review Letters, 112, 215002

Paschmann, G., \& Sonnerup, B. U. O. 2008, ISSI Scientific Reports Series, 8, 65

Zank, G. P., Adhikari, L., Hunana, P.,
Servidio, S., Matthaeus, W. H., \& Dmitruk, P. 2008, Physical Review Letters, 100, 095005 2016, ApJ, 826, 205

Telloni, D., Perri, S., Bruno, R., Carbone,

Vasquez, B. J., Abramenko, V. I., Haggerty, D. K., \& Smith, C. W. 2007, Journal of Geophysical Research (Space Physics), 112, A11102

Wan, M., Matthaeus, W. H., Roytershteyn, V., et al. 2015, Physical Review Letters, 114, 175002

Wan, M., Matthaeus, W. H., Servidio, S., \& Oughton, S. 2013, Physics of Plasmas, 20, 042307

Yu, W., Farrugia, C. J., Lugaz, N., et al. 2014, J. Geophys. Res., 119, 689 et al. 2017, The Astrophysical Journal, $835,147$. http://stacks.iop.org/0004-637X/835/i=2/a=147

Zhao, L.-L., Adhikari, L., Zank, G. P., Hu, Q., \& Feng, X. S. 2017, ApJ, 849, 88

—. 2018, ApJ, in preparation

Zheng, J. 2017, PhD thesis, The University of Alabama in Huntsville 